\title{
Qualidade de vida de pacientes renais crônicos submetidos à fisioterapia na hemodiálise
}

\author{
Quality of life of chronic renal patients submitted to physiotherapy during hemodialysis
}

\author{
Clarissa Rios Lara ${ }^{1}$, Fernanda Agnys Oliveira Guirra Santos ${ }^{1}$, Thelso de Jesus Silva ${ }^{2}$ \\ Fernanda Warken Rosa Camelier ${ }^{3}$
}

\begin{abstract}
RESUMO
Objetivo: Avaliar a qualidade de vida de um grupo de pacientes com Insuficiência Renal Crônica antes e após a realização de um programa de fisioterapia durante a hemodiálise.

Materiais e Métodos: Trata-se de um ensaio clínico não controlado, do tipo antes e depois, onde a qualidade de vida foi mensurada através do questionário Medical Outcomes Study 36 - Item Short-Form Health Survey (SF-36), antes e após um programa de exercícios físicos direcionados à pacientes submetidos à hemodiálise. Foram realizadas 24 sessões de fisioterapia durante oito semanas, com duração de 25 minutos, sendo incluídos exercícios de alongamentos, fortalecimento muscular, consciência respiratória e relaxamento. Foi utilizado o teste T pareado para comparação dos resultados. Quanto às diferenças clinicamente relevantes, foram considerados um aumento de cinco ou mais pontos para os domínios do SF-36, e de dois e meio ou mais pontos para os componentes Physical Components Summary (PCS) e Mental Components Summary (MCS).

Resultados: Para os 17 participantes deste estudo, apenas o domínio Capacidade Funcional $(p=0,04)$ e os componentes Physical Components Summary $(p=0,01)$ e Mental Components Summary $(p=0,04)$ apresentaram relevância estatística. Em relação à diferença clinicamente relevante, os domínios Capacidade Funcional, Aspectos Físicos, Vitalidade, Aspectos Sociais e Aspectos Emocionais foram considerados (maior do que 5 pontos), assim como os componentes Physical Components Summary e Mental Components Summary (maior do que 2,5 pontos).

Conclusão: Este estudo mostrou que a assistência fisioterapêutica realizada durante a hemodiálise proporcionou melhora em algumas dimensões na qualidade de vida do grupo estudado, principalmente no domínio Capacidade Funcional, onde se obteve aumento estatisticamente significativo e melhora clinicamente relevante.
\end{abstract}

Palavras-chave: qualidade de vida; insuficiência renal crônica; diálise renal; fisioterapia.

\section{ABSTRACT}

Objective: To evaluate the quality of life in a group of patients with Chronic Renal Disease before and after a physiotherapy program during hemodialysis.

Materials and Methods: This is a non controlled, before and after, clinical trial where the quality of life was measured using the questionary Medical Outcomes Study 36 - Item Short-Form Health Survey (SF-36), before and after a program of physical activities directed to patients submitted to hemodialysis. Twenty four sessions of physiotherapy were performed during eight weeks. The paired t-test was used for comparison of the results. For analyses of the relevant clinical differences, an increase of five or more points was considered for the domains of SF-36, and of 2.5 or more points for the components of Physical Components Summary (PCS) and Mental Components Summary (MCS).

Results: For the 17 participants of this study, only the domain Functional Capacity $(p=0.04)$ and the components Physical Components Summary $(p=0.01)$ and Mental Components Summary $(p=0.04)$ presented statistical relevance. In relation to the clinically relevant difference, the domains Functional Capacity, Physical Aspects, Vitality, Social Aspects and Emotional Aspects were considered (higher than 5 points), even as the components of Physical Components Summary and Mental Components Summary (higher than 2.5 points).

Conclusions: This study suggests that physiotherapeutic assistance, performed during hemodyalisis, provided improvements in some dimensions of quality of life of the studied group, mainly functional capacity, where we found statistically significant and clinically relevant improvement.

Keywords: quality of life; chronic renal insufficiency; renal dialysis, physical therapy specialty.

\footnotetext{
${ }^{1}$ Fisioterapeuta pela Universidade Católica de Salvador (UCSal). Hospital São Rafael, Salvador (BA).

${ }^{2}$ Fisioterapeuta. Mestre em Medicina e Saúde pela Universidade Federal da Bahia (UFBA). Hospital São Rafael, Salvador (BA).

${ }^{3}$ Fisioterapeuta. Doutora em Ciências/Reabilitação pela Universidade Federal de São Paulo (Unifesp).
} 


\section{INTRODUÇÃO}

A Insuficiência Renal Crônica (IRC) é a perda irreversível da função renal, caracterizada por diminuição da taxa de filtração glomerular, geralmente decorrente de um processo patológico lentamente progressivo. Esta condição interfere no equilíbrio metabólico, hidroeletrolítico e ácido-básico do organismo, altera os padrões normais de diurese, culmina em uremia e acomete quase todos os sistemas $\operatorname{orgânicos}^{1,2}$. As principais causas da IRC são glomerulopatia primária, pielonefrites, Hipertensão Arterial Sistêmica (HAS) e Diabetes Mellitus ${ }^{1}$. A perda da função renal representa risco à vida e requer a remoção de resíduos tóxicos e restauração do volume e composição dos líquidos corpóreos e isso pode ser feito por meio da diálise ${ }^{3}$. Dentre os tratamentos de substituição ou dialíticos indicados estão à hemodiálise (HD), que é a terapia mais comumente realizada, e o transplante renal, o qual tem obtido melhores resultados ${ }^{1}$.

A IRC é considerada um grande problema de saúde pública porque causa elevadas taxas de morbimortalidade e tem impacto negativo sobre a qualidade de vida ${ }^{4}$. Atualmente, no Brasil, existem 92.091 pacientes em terapia renal substitutiva, conforme o Censo de Diálise de 2010, sendo 90,6\% em hemodiálise e o restante em diálise peritonial, e 38,7\% na fila de espera para transplante renal ${ }^{5}$.

O tratamento hemodialítico torna ainda mais problemática a qualidade de vida do paciente, pois limita as atividades e favorece o sedentarismo e a deficiência funcional. A monotonia e dependência associadas às restrições dietéticas e hídricas, a presença de cateteres e fístulas arteriovenosas provocam alterações na capacidade física, auto-estima, imagem corporal do paciente ${ }^{6,7}$ e compromete o aspecto físico e psicológico, com repercussões pessoais, familiares e sociais ${ }^{7}$. Estas mudanças biopsicossociais trazem agravantes como a preocupação com o número insuficiente de rins disponíveis para transplante, o isolamento social, perda do emprego, dependência da previdência social, medo de morrer e outros ${ }^{7,8}$.

A prática de atividade física tem efeitos positivos sobre a saúde mental. A literatura aponta os benefícios do exercício físico sobre a área emocional pela obtenção de melhoras significativas nos níveis de depressão, auto-imagem, ansiedade, sono, humor, vivacidade mental, controle de peso, senso de responsabilidade com a própria saúde, além de ser uma forma alternativa e eficaz de liberar tensões, emoções e frustrações $^{9}$, características do paciente com IRC. Estudos mostraram que a realização de exercícios durante a diálise pode promover a motivação do doente em um ambiente estruturado e monótono ${ }^{10,11}$. Foi observado que após o programa de fisioterapia no período hemodialítico houve melhora significativa nas dimensões capacidade funcional, dor, vitalidade, saúde mental e no desempenho nas atividades diárias e de trabalho, apesar da sensação de desânimo, cansaço e falta de energia, sintomas frequentes em pacientes renais crônicos ${ }^{12}$. Corrêa et al. ${ }^{13}$, em um estudo de cinco meses usando treinamento muscular periférico em um grupo de 18 pacientes, demonstraram que após a intervenção fisioterapêutica houve aumento dos valores das dimensões aspectos físicos, dor, estado geral de saúde e vitalidade. Em outros estudos, houve aumento significativo no domínio vitalidade ${ }^{14}$, e $\operatorname{dor}^{15}$ em pacientes que foram submetidos a treinamento físico durante a HD.

Apesar de ser um tema de relevância atual, poucos centros de hemodiálise oferecem programas de exercício ou acompanhamento com fisioterapeutas aos pacientes submetidos à hemodiálise, justificando a importância de ampliar a atuação da fisioterapia em toda essa clientela e desenvolver estudos que avaliem a eficácia deste tratamento. Os resultados das pesquisas demonstram a necessidade de acompanhar diferentes grupos por um período de tempo extenso, com grande amostra e inclusão de grupo controle, com o objetivo de demonstrar os prováveis benefícios da fisioterapia no período transdialítico ${ }^{15}$. O objetivo do presente estudo foi avaliar a qualidade de vida antes e após a realização de um programa de fisioterapia em um grupo de pacientes submetidos à hemodiálise.

\section{MATERIAIS E MÉTODOS}

Trata-se de um ensaio clínico não controlado, do tipo antes e depois, onde a qualidade de vida foi mensurada 
através do questionário SF-36, aplicado antes e após um programa de exercícios físicos direcionados à pacientes com IRC submetidos à hemodiálise na unidade de nefrologia de um hospital situado na cidade de Salvador, Bahia. Foram selecionados pacientes que realizavam HD três vezes por semana em determinados dias e turnos, com faixa etária maior que 18 anos, em tratamento ambulatorial no programa de hemodiálise da instituição há mais de 12 semanas, sem déficit cognitivo, e que concordaram em participar do estudo mediante a assinatura do Termo de Consentimento Livre e Esclarecido (TCLE). Foram excluídos os pacientes de outros turnos que esporadicamente realizavam HD com o grupo estudado, com incapacidade de colaboração ou entendimento do questionário de qualidade de vida SF36, déficit motor como amputação, paralisias ou paresias, ou que realizavam fisioterapia fora da HD.

Foi utilizado um formulário elaborado pelos autores para a coleta de dados sociodemográficos e clínicos com a finalidade de caracterizar os sujeitos quanto à idade, estado civil, escolaridade, ocupação, tempo de tratamento, prática de atividades físicas, diagnóstico clínico, co-morbidades, avaliação funcional e exame físico. Também foram utilizados os prontuários dos pacientes como fonte secundária na coleta de dados.

A qualidade de vida foi avaliada por meio da aplicação do questionário genérico SF-36 - Medical Outcomes Study 36 - Item Short-Form Health Survey ${ }^{16}$, traduzido e validado no Brasil por Ciconelli ${ }^{17}$, composto por 36 itens que avaliam os componentes saúde física e saúde mental, com os seguintes domínios: capacidade funcional, aspectos físicos, dor, estado geral de saúde, vitalidade, aspectos sociais, aspectos emocionais e saúde mental. Os resultados de cada componente variam de 0 a 100 , sendo 0 (zero) o mais comprometido e 100 (cem) nenhum comprometimento.

No sentido de reduzir o número de medidas de resultado em saúde e melhorar a sua interpretação, foram extraídos dois componentes dois oito domínios: o resumo dos componentes físico (Physical Components Summary - PCS) e mental (Mental Components Summary - MCS), que expressam os agrupamentos dos componentes físico (capacidade funcional, aspectos físicos, dor e estado geral de saúde) e componente mental (vitalidade, aspectos sociais, aspectos emocionais e saúde mental) do questionário, respectivamente. Estas duas componentes foram desenvolvidas no decorrer de estudos de avaliação das propriedades psicométricas do SF-36, verificando-se que estas medidas capturavam 80 a $85 \%$ da variância dos oito domínios, ficando assim, a análise e interpretação do SF-36 simplificada ${ }^{16,18}$. Foi obtido um termo de consentimento para o uso deste questionário, mediante assinatura da QualityMetric ${ }^{19}$. A aplicação do instrumento foi realizada durante a HD, antes e após a intervenção fisioterapêutica, para então serem comparados os resultados obtidos.

O atendimento fisioterapêutico foi realizado nas primeiras horas do tratamento hemodialítico, respeitando a condição clínica do participante, com duração de 25 minutos cada sessão, três vezes na semana, no período de oito semanas. Os exercícios eram realizados na posição de decúbito dorsal ou sedestração, em uma poltrona inclinável. Ao início do atendimento fisioterapêutico, eram realizados alongamentos de 20 segundos, seguido de exercícios metabólicos e exercícios de resistência muscular para membros superiores e inferiores com halter ou caneleira de um quilograma. Ao final da sessão, eram propostos exercícios de relaxamento envolvendo a conscientização respiratória. A descrição do protocolo fisioterapêutico utilizado está descrito na tabela 1.

A situação hemodinâmica do paciente foi investigada antes e após a terapia, aferindo-se a pressão arterial e a frequência cardíaca. $O$ paciente foi dispensado da fisioterapia quando apresentava quadro álgico muito importante, tonturas, hipertensão ou hipotensão acentuadas. Não foi mobilizado no membro o segmento cujo acesso venoso se encontrava, para evitar risco de comprometimento.

Os dados dos questionários foram digitados em uma planilha no software Microsoft Excel, e a análise dos mesmos foi realizada por meio do software estatístico SPSS, versão 17.0. As variáveis categóricas estão descritas em proporções e as quantitativas apresentadas em média e desvio padrão. Para a análise da normalidade foi realizado o teste de KolmogorovSmirnov. O teste T pareado foi utilizado para comparar a 
TABELA 1 - Descrição do protocolo fisioterapêutico aplicado.

\begin{tabular}{|c|c|}
\hline $\begin{array}{c}\text { Alongamentos } \\
\text { (20 segundos para cada segmento) }\end{array}$ & $\begin{array}{ll}\text { - } & \text { Quadríceps } \\
\text { - } & \text { Isquiotibiais e tríceps sural } \\
\text { - } & \text { Adutores e abdutores do quadril } \\
\text { - } & \text { Coluna lombar: dissociação de cinturas } \\
\text { - } & \text { Coluna cervical: escalenos, trapézio superior, suboccipitais, } \\
\text { - } & \text { Deltóide superior } \\
\text { - } & \text { Tríceps } \\
\text { - } & \text { Peitoral maior } \\
\text { - } & \text { Flexores e extensores do punho }\end{array}$ \\
\hline $\begin{array}{l}\text { Exercícios circulatórios } \\
\text { (10 repetições para cada exercício) }\end{array}$ & $\begin{array}{ll}\text { - } & \text { Bombeio de gastrocnêmios } \\
\text { - } & \text { Circundução de punho e ombro } \\
\text { - } & \text { Flexão e extensão de quirodáctilos }\end{array}$ \\
\hline $\begin{array}{c}\text { Cinesioterapia resistida } \\
\text { (duas séries de } 10 \text { repetições) }\end{array}$ & $\begin{array}{ll}\text { - } & \text { Elevação da perna estendida } \\
\text { - } & \text { Flexão e extensão de joelhos } \\
\text { - } & \text { Abdução e adução de quadril } \\
\text { - } & \text { Elevcundução de quadril com perna estendida } \\
\text { - } & \text { Flexão e extensão de ombro e cotovelo } \\
\text { - } & \text { Abdução e adução de ombro }\end{array}$ \\
\hline $\begin{array}{l}\text { Conscientização respiratória } \\
\text { (10 repetições) }\end{array}$ & $\begin{array}{l}\text { - Padrões ventilatórios reexpansivos: inspiração profunda, } \\
\text { soluços inspiratórios, sustentação máxima da inspiração } \\
\text { - Freno labial }\end{array}$ \\
\hline
\end{tabular}

pontuação dos domínios e componentes do questionário pré e pós intervenção. Um $p<0,05$ foi considerado estatisticamente relevante. Para análise das diferenças clinicamente relevantes foram considerados um aumento de cinco ou mais pontos para os domínios do SF-36, e aumento de dois e meio ou mais pontos para os componentes PCS e MCS M,21. $^{20}$.

Conforme a resolução 196/96 do CONEP, o trabalho foi realizado após aprovação do Comitê de Ética e Pesquisa do Hospital São Rafael (HSR) pelo Parecer 57325/12, e após consentimento formal e esclarecido dos pacientes, registrado em formulário próprio no Termo de Consentimento Livre e Esclarecido. Os participantes do estudo foram comunicados que poderiam interromper sua participação em qualquer etapa da pesquisa, sem prejuízo para sua pessoa. Não existe indicação de que os procedimentos do estudo ofereceram riscos. A natureza voluntária da participação e os procedimentos de garantia de confidencialidade e anonimato dos dados foram descritos para os participantes.

\section{RESULTADOS}

Foram selecionados 20 pacientes, destes três foram excluídos, um por realizar fisioterapia domiciliar semanalmente, e dois por solicitarem suspensão do tratamento, totalizando 17 pacientes. O período de intervenção fisioterapêutica a que o grupo estudado foi submetido cumpriu as oito semanas previstas, com um total de 24 sessões de fisioterapia realizadas para cada um dos indivíduos do estudo.

Do total de 17 participantes, 14 eram do sexo masculino (82,4\%). A média de idade de todos foi de $55,2 \pm 17,0$ anos. Quanto ao estado civil, 12 são casados. Em relação à ocupação, 11 pacientes são aposentados. O tempo de hemodiálise variou de três meses a 13 anos, sendo a média de $5,9 \pm 4,8$ anos. Todos os pacientes apresentavam pelo menos uma co-morbidade como Hipertensão arterial sistêmica, Diabetes Mellitus, anemia, cardiopatia e gota. A hipertensão arterial foi mais frequente, pois esteve presente em $94,1 \%$ dos participantes. Quanto à atividade física, 14 são sedentários. As características sociodemográficas da população estudada foram agrupadas na tabela 2 .

A tabela 3 representa a análise dos domínios e componentes relacionados ao questionário de vida SF36 na pré e pós intervenção fisioterapêutica. Todas as médias mostraram aumento após o treinamento, quando comparada ao período pré intervenção, exceto os domínios Estado Geral de Saúde e Dor. Apenas o 
TABELA 2 - Características sociodemográficas dos pacientes com Insuficiência Renal Crônica estudados no HSR.

\begin{tabular}{lc}
\hline Variáveis & $\mathbf{n}(\%)$ \\
\hline Sexo & $14(82,4)$ \\
Masculino & $3(17,6)$ \\
Feminino & \\
Estado civil & $12(70,6)$ \\
Casado & $4(23,5)$ \\
Solteiro & $1(5,9)$ \\
Viúvo & \\
Escolaridade & $5(29,4)$ \\
Fundamental & $6(35,3)$ \\
Ensino médio & $6(35,3)$ \\
Ensino Superior & \\
Prática de atividade física & $3(17,6)$ \\
Sim & $14(82,4)$ \\
Não & \\
Ocupação & $11(64,7)$ \\
Aposentados & $6(35,2)$ \\
Trabalhando & \\
Co-morbidades & $16(94,1)$ \\
HAS & $3(17,6)$ \\
DM & $3(17,6)$ \\
Gota & $11(64,7)$ \\
Tempo de Hemodiálise & $6(35,2)$ \\
$<10$ anos & \\
IMC anos & $1(5,9)$ \\
Abaixo do peso & \\
Peso normal & \\
Sobrepeso & \\
\hline & \\
\hline & \\
\hline
\end{tabular}

domínio Capacidade Funcional, que alcançou média inicial de $46,8 \pm 27,3$, e final de $58,5 \pm 25,7$, foi considerado estatisticamente relevante, pois apresentou $p=0,04$. Foi observado significância estatística também nos componentes PCS e MCS. O PCS, que resume o componente físico, teve média inicial de $35,9 \pm 7,8$ e ao final do programa $39,2 \pm 31,6$, com $p=0,01$. Enquanto que o escore MCS que resume o componente mental teve média inicial de $45,2 \pm 23,2$ e ao final do programa $49,8 \pm 25,9$, com $p=0,04$.

Ao analisar as diferenças entre a média inicial e final de cada domínio, constatou-se que cinco de oito domínios apresentaram diferença $(\Delta)$ maior que cinco pontos, o que é considerado clinicamente relevante. Os cinco domínios são Aspectos Físicos $(\Delta 11,8)$, Vitalidade
$(\Delta 5,9)$, Aspectos Sociais $(\Delta 8,3)$, Aspectos Emocionais $(\Delta 19,5)$ e Capacidade Funcional $(\Delta 11,8)$. Os componentes PCS $(\Delta 11,5)$ e MCS $(\Delta 13,0)$ foram considerados como clinicamente relevantes ( $\Delta \geq$ dois e meio pontos).

\section{DISCUSSÃo}

O presente estudo mostrou que a realização de um programa de exercícios físicos durante a hemodiálise pode promover a melhora da qualidade de vida de pacientes renais crônicos nos aspectos capacidade funcional, aspectos físicos, vitalidade, aspectos sociais e aspectos emocionais, além de aumentos nos componentes PCS e MCS. Estes escores apresentaram diferenças clinicamente relevantes e alguns deles importância estatística quando comparamos as suas médias nos períodos pré e pós intervenção fisioterapêutica.

A presença de uma doença crônica está associada à piora da qualidade de vida de uma população ${ }^{22}$. Dentre os estudos que abordaram a questão da qualidade de vida de pessoas com IRC submetidas a tratamento hemodialítico, Cunha et al. $^{23}$ encontraram uma perspectiva negativa de viver com esta condição, sendo relatado pelos pacientes a percepção do impacto da doença em sua qualidade de vida, que traz importantes limitações físicas, psicológicas e sociais. Várias condições podem interferir nesta percepção, tais como: idade, tempo de tratamento, presença de comorbidades, intercorrências recentes durante tratamento dialítico, baixo condicionamento físico e anemia, que são condições bastante frequentes na população estudada e podem explicar a inatividade física e baixa qualidade de vida 22,23 .

Tem crescido o interesse dos profissionais da saúde em mensurar a qualidade de vida destes pacientes, pois existe uma relação direta entre o nível de qualidade de vida e a sobrevida ${ }^{12}$. Os tratamentos dialíticos não chegam a substituir integralmente a função renal, mas representam condições para manter a sobrevida da pessoa, permitem que esta retorne a uma vida normal e produtiva e previnem a morte precoce ${ }^{2}$. Os avanços tecnológicos e terapêuticos na área de diálise contribuíram para o aumento da sobrevida dos renais 
TABELA 3 - Valores da pontuação para cada domínio e componentes do questionário SF-36 antes e após a intervenção fisioterapêutica nos participantes da hemodiálise do HSR.

\begin{tabular}{lccccccc}
\hline \multicolumn{7}{c}{ Pré-intervenção } & \multicolumn{2}{c}{ Pós-intervenção } & & & \\
\hline Domínios SF-36 & Média \pm DP & Amplitude & Média \pm DP & Amplitude & p & $\Delta \pm$ DP & Mín - Máx \\
Capacidade funcional & $46,8 \pm 27,3$ & $5-85$ & $58,5 \pm 25,7$ & $5-95$ & 0,04 & $11,8 \pm 14,4$ & $4,4-19,1$ \\
Aspecto físico & $27,9 \pm 32,9$ & $0-100$ & $39,7 \pm 45,1$ & $0-100$ & 0,303 & $11,8 \pm 49,3$ & $-13,6-37,1$ \\
Dor & $56,5 \pm 24,2$ & $22-100$ & $51,9 \pm 26,6$ & $10-100$ & 0,649 & $-4,6 \pm 28,7$ & $-19,4-10,2$ \\
Estado Geral de Saúde & $59,9 \pm 20,3$ & $32-92$ & $58,3 \pm 27,7$ & $0-100$ & 0,755 & $-1,6 \pm 23,6$ & $-13,7-10,5$ \\
Vitalidade & $52,4 \pm 16,1$ & $25-80$ & $58,2 \pm 24,1$ & $0-100$ & 0,419 & $5,9 \pm 27,6$ & $-8,3-20,1$ \\
Aspecto social & $64,0 \pm 26,1$ & $12,5-100$ & $72,3 \pm 28,1$ & $0-100$ & 0,163 & $8,3 \pm 26,5$ & $-5,4-21,9$ \\
Aspecto emocional & $47,1 \pm 42,6$ & $0-100$ & $66,9 \pm 39,2$ & $0-100$ & 0,255 & $19,5 \pm 60,3$ & $-11,5-50,5$ \\
Saúde mental & $69,9 \pm 14,1$ & $44-92$ & $74,1 \pm 23,4$ & $8-100$ & 0,204 & $4,2 \pm 17,3$ & $-4,7-13,2$ \\
Componentes SF-36 & & & & & & & $11,5 \pm 9,1$ \\
PCS & $35,9 \pm 7,8$ & $26,5-52,2$ & $47,4 \pm 6,2$ & $39,2-58,5$ & 0,01 & $6,8-16,2$ \\
MCS & $36,9 \pm 10,5$ & $21,0-52,6$ & $49,9 \pm 12,3$ & $14,0-63,1$ & 0,04 & $13,0 \pm 13,4$ & $6,1-19,9$ \\
\hline
\end{tabular}

Amplitude: mínimo - máximo; DP: desvio-padrão; $p$ : nível de significância estatística $(p<0,05) ; \Delta$ : média da diferença dos escores de qualidade de vida pós x pré intervenção; Mín - Max: valores mínimo e máximo da diferença entre média da diferença dos escores de qualidade de vida pós x pré intervenção; PCS: resumo do componente físico do SF-36 (capacidade funcional, aspectos físicos, dor e estado geral de saúde); MCS: resumo do componente mental do SF-36 (vitalidade, aspectos sociais, aspectos emocionais e saúde mental).

crônicos, sem, no entanto, possibilitar-lhes a melhora da qualidade de $v_{i d a}{ }^{15}$, pois a permanência por tempo indeterminado em tratamento dialítico pode interferir nesta condição ${ }^{22}$.

A IRC traz consequências para quase todos os sistemas do corpo humano, dentre eles o sistema muscular, que pode sofrer atrofia muscular, miopatia e má nutrição. Esta situação ocasiona fraqueza generalizada no organismo, diminuição na tolerância ao exercício físico, descondicionamento físico progressivo e fadiga dos pacientes submetidos a tratamento hemodialítico ${ }^{13,23}$. É conhecida a importância da prática regular de exercício físico para a manutenção da saúde e qualidade de vida devido à sua contribuição na prevenção de várias morbidades, além do aumento na autoestima e redução da depressão e isolamento social ${ }^{24}$.

A doença renal crônica afeta na sua maioria a população mais idosa, associada à co-morbidades, e, em menor escala, pacientes mais jovens, em idade produtiva, causando implicações médicas, sociais e econômicas $^{15}$. O presente estudo observou uma correlação negativa entre a idade avançada e as dimensões capacidade funcional, aspectos físicos e aspectos emocionais. Semelhante a esses achados, Soares et al. ${ }^{12}$, ao aplicarem o SF-36 em 27 pacientes em diálise, observaram as mesmas correlações. Já
Guedes et al. ${ }^{25}$, observaram que com o avançar da idade houve maior comprometimento das atividades físicas e funcionais dos pacientes, enquanto o componente emocional foi menos afetado.

O tempo de tratamento hemodialítico correlacionou-se negativamente com os aspectos emocionais na amostra aqui estudada, sugerindo que pacientes com maior tempo de tratamento dialítico apresentam comprometimento das relações familiares e sociais. Por outro lado, aspectos emocionais correlacionaram-se positivamente com anos de estudo, sugerindo que alguns dos pacientes com maior escolaridade podem possuir recursos intelectuais capazes de gerar melhor adaptação emocional às consequências da doença renal crônica e do tratamento. Quanto mais o paciente renal em hemodiálise estiver informado sobre sua doença e tratamento, melhor será sua qualidade de vida. Em recente revisão, Guedes et al. ${ }^{25}$ relataram que a piora da qualidade de vida correlacionou-se com maior tempo de tratamento hemodialítico e o avançar da idade, corroborando com os dados encontrados na presente pesquisa.

Em estudo realizado por Trentini et al. ${ }^{7}$, pessoas com IRC submetidas a tratamento hemodialítico ganham peso entre uma sessão e outra de hemodiálise devido à retenção de líquido, o que vem a causar cansaço, entre outras alterações, prejudicando assim a capacidade 
funcional. Esses achados foram observados no presente estudo, pois o subgrupo com sobrepeso apresentou menor escore médio no domínio aspectos físicos do SF36 quando comparado ao grupo com IMC normal ou menor.

Hipertensão arterial sistêmica (HAS) e Diabetes Mellitus (DM) são as importantes causas de doença renal. A DM é responsável por um terço dos pacientes que iniciam programa de substituição renal, enquanto que 10 a $15 \%$ dos pacientes hipertensos desenvolvem insuficiência renal crônica ${ }^{15}$. A hipertensão arterial esteve presente em 16 pacientes deste estudo, enquanto a diabetes foi encontrada em três participantes da pesquisa. Este dado corrobora com o estudo de Marques et al. ${ }^{2}$, que apontam maior prevalência dos pacientes renais crônicos com patologia de base HAS.

Os pacientes com IRC queixam-se frequentemente de desconforto e/ ou dor, principalmente nas regiões lombar e cervical. Esse fato pode ser explicado por vários motivos, como: presença de distúrbios musculoesqueléticos, permanência na posição "sentado" e "estático", má postura do paciente ao sentar e cadeiras padronizadas que, muitas vezes, não respeitam as individualidades ergonômicas de cada paciente ${ }^{12}$.

A fraqueza muscular é uma complicação frequente da IRC, logo, pode-se afirmar a importância dos exercícios de fortalecimento para minimizar essa perda de massa muscular, além de promover a força necessária para que o indivíduo exerça suas atividades ${ }^{12}$. No presente estudo, verificou-se melhora clinicamente relevante dos aspectos emocionais dos participantes, assim como o estudo de Cunha et al. ${ }^{23}$. Provavelmente, quando o indivíduo consegue realizar suas atividades de vida diária (AVD) com menor esforço ou dor, sente-se menos cansado e mais motivado.

Soares et al. ${ }^{12}$, relatam que a taxa de aderência dos pacientes ao programa de reabilitação, durante a hemodiálise, é muito maior quando comparada a do programa ambulatorial, provavelmente porque, quando realizado durante a diálise, o exercício não tome tanto tempo como um programa formal realizado em outro turno. Além da adesão ao programa de fisioterapia aplicado durante a hemodiálise, observamos que, no grupo estudado, os pacientes se mostraram receptivos e colaborativos na realização das atividades propostas, o que sugere a boa aceitação da fisioterapia neste contexto. Quando um indivíduo apresenta diagnóstico de insuficiência renal, costuma seguir-se um longo período de restrição da atividade física, e esta inatividade leva a um progressivo descondicionamento que limita ainda mais a capacidade física ${ }^{15}$. Neste estudo, três pacientes descreveram atividade física como rotina antes do advento da doença renal crônica, mas quatro participantes da pesquisa que realizavam algum tipo de atividade física antes da doença abandonaram totalmente este hábito, destacando-se a necessidade de resgatá-lo. Painter ${ }^{11}$ desenvolveu um projeto com pacientes portadores de DRC em hemodiálise, com exercícios metabólicos, de alongamentos, fortalecimentos e exercícios aeróbicos, prescritos individualmente para serem realizados durante a hemodiálise. Este autor concluiu que a realização de exercícios físicos durante a hemodiálise ocasiona ganho na capacidade física e melhor qualidade de vida para os pacientes com doença renal crônica. Esta relação também foi constatada nesta pesquisa, pois o domínio capacidade funcional apresentou melhora significativa após o programa de fisioterapia. $\mathrm{Na}$ avaliação inicial, constatou-se comprometimento de algumas dimensões analisadas e o menor valor foi em aspectos físicos, o que já foi indicado pela literatura ${ }^{12}$. Soares et al. ${ }^{12}$ verificaram que após o tratamento, houve aumento de todas as médias e melhora significativa das variáveis: capacidade funcional, dor, vitalidade, saúde mental e o desempenho nas atividades diárias e de trabalho, apesar da sensação de desânimo, cansaço e falta de energia, os quais são sintomas frequentes em pacientes renais crônicos.

Os resultados obtidos no estudo de Corrêa et al. ${ }^{13}$ demonstraram que as médias, que aumentaram após o treinamento muscular periférico, foram os valores das dimensões aspectos físicos, dor, estado geral de saúde e vitalidade, porém não apresentaram significância. No entanto, um estudo de Torkington et al. ${ }^{14}$ mostrou que os pacientes submetidos a treinamento físico 
apresentam resultados significativos no domínio vitalidade. Os resultados do estudo de Castro et al. ${ }^{21}$ evidenciaram comprometimento nas diferentes dimensões analisadas pelo questionário SF-36, sendo que os menores valores médios foram observados nas dimensões aspectos físicos e vitalidade. Já Peres et al. ${ }^{26}$ observaram melhora significativa na capacidade funcional, vitalidade, aspectos emocionais, saúde mental, aspectos físicos e dor. Cunha et al. ${ }^{23}$, verificaram que no SF-36, as pontuações foram mais elevadas nos domínios aspecto social e aspectos emocionais; e mais baixas foram nos domínios dor e vitalidade. No estudo de Martins \& Cesarino ${ }^{4}$ foram observados menores escores nos domínios dos aspectos físicos, emocionais e vitalidade. No estudo de Soares et al. ${ }^{15}$, observou-se melhora nas dimensões aspectos físicos, vitalidade, estado geral de saúde, aspecto social, e maior escore no domínio dor, porém sem significância estatística.

No presente estudo, verificou-se melhora estatisticamente significativa no domínio Capacidade Funcional, o que está de acordo com os estudos de Soares et al. $^{12}$ e Peres et al. ${ }^{26}$, indicando provável menor gasto energético nas AVD's e maior independência funcional ao final do período de intervenção. Foi encontrada também relevância significativa nos resumos do componente físico e mental, o PCS e MCS, indicando que houve aumento geral dos oito domínios do SF-36, e desta forma, sugerindo fortemente a melhora da qualidade de vida de pacientes em hemodiálise associada à prática de exercícios físicos. Estes dados não foram encontrados pelos autores em outros estudos semelhantes. $O$ fato de não ter sido obtido aumento significativo nos outros componentes provavelmente tem relação com o curto período de tratamento fisioterapêutico e número reduzido de participantes no estudo. Em relação às diferenças clinicamente relevantes, foi observado melhora nos cinco domínios Capacidade Funcional, Aspectos Físicos, Vitalidade, Aspectos Sociais e Aspectos Emocionais, além dos componentes PCS e MCS. Estas informações sugerem que, apesar dos quatro últimos domínios não apresentarem relevância estatística, foram considerados importantes na prática clínica. Outras pesquisas similares não foram encontradas para comparação de dados.

As percepções negativas e desmotivação pessoal muitas vezes foram encontradas nestes pacientes. A restrição imposta pela hemodiálise, principalmente quanto ao manuseio do segmento onde se encontra o acesso venoso, e o uso de exercícios localizados como única estratégia de tratamento também foram considerados fatores limitantes do estudo. No entanto, observamos que o exercício físico realizado no período transdialítico mostrou-se uma prática segura e bem aceita pelos pacientes, agindo também como fator motivacional.

Outras limitações encontradas foram o pequeno tamanho amostral, tempo de tratamento e a necessidade de inclusão de grupo controle, porém este estudo foi realizado em caráter experimental na instituição selecionada, onde não havia o atendimento fisioterapêutico no setor de Hemodiálise do hospital. Os autores persistem em investigar e aprofundar em novos estudos sobre o tema, que possui importante relevância científica, com estes pontos contemplados. Embora existam evidências sobre os benefícios do exercício, muitos pacientes permanecem inativos, pois esses programas normalmente não oferecidos nos serviços de hemodiálise. Esse fato permite sugerir que a inclusão de fisioterapeutas nos centros de hemodiálise deve ser avaliada, já que a realidade na maioria desses centros não oferece esse tipo de serviço ao paciente, muitas vezes justificando-se pelos riscos adversos dos exercícios. Esta pesquisa foi de baixo custo, fácil aplicabilidade e recomenda a atuação da fisioterapia no serviço de hemodiálise, pela possibilidade de melhora da qualidade de vida de pacientes renais crônicos, conforme verificado nesta amostra. Além disso, contribuiu para o acervo de estudos existentes acerca do tema e buscou detalhar o protocolo fisioterapêutico utilizado.

Este estudo mostrou que a fisioterapia, quando realizada durante a hemodiálise, proporcionou melhora da qualidade de vida destes, como verificado no aumento do escore de alguns domínios do questionário SF-36. 


\section{REFERÊNCIAS}

1. Engel CL, Marinho ML, Durand A, Engel H, Rocha M. Nefrologia. São Paulo: Medbros Editora; 2005. v.5.

2. Marques AB, Pereira DC, Ribeiro RCHM. Motivos e frequência de internação dos pacientes com IRC em tratamento hemodialítico. Arq Ciênc Saúde 2005;12(2): 67-72.

3. Guyton AC, Hall JE. Os Líquidos Corpóreos e os Rins. In: Guyton AC, Hall JE. Tratado de Fisiologia Médica. Brasil: Editora Elsevier, 2006, p. 404-15.

4. Martins MRI, Cesarino CB. Qualidade de vida de pessoas com doença renal crônica em tratamento hemodialítico. Rev Latino-am Enfermagem 2005; 13(5):670-6.

5. Sesso RC, Lopes AA, Thomé FS, Lugon JR, Santos DR. Relatório do Censo Brasileiro de Diálise de 2010. J Bras Nefrol. 2011; 33(4):442-7.

6. Coelho DM, Castro AM, Tavares HA, Abreu PBC, Glória RR, Duarte MH, Oliveira MR. Efeitos de um programa de exercícios físicos no condicionamento de pacientes em hemodiálise. J Bras Nefrol 2006; 28(3):121-7.

7. Trentini M, Corradi EM, Araldi MAR, Tigrinho FC. Qualidade de vida de pessoas dependentes de hemodiálise considerando alguns aspectos físicos, sociais e emocionais. Rev Texto-Contexto Enferm. 2004; 13(1): 74-82.

8. Carreira L, Marcon SS. Cotidiano e trabalho: concepções de indivíduos portadores de insuficiência renal crônica e seus familiares. Rev Latino-Am Enfermagem. 2003; 11: 823-31.

9. Godoy RF. Benefícios do exercício físico sobre a área emocional. Movimento. 2002; 8(2):7-16.

10. Knap B, Ponikvar JB, Ponikvar R, Bren AF. Regular exercise as a part of treatment for patients with endstage renal disease. Ther Apher Dial 2005; 9(3):211-3.

11. Painter $P$. Low-Functioning Hemodialysis Patients Improve With Exercise Training. Am J Kidney Dis. 2000;36(3):6008.

12. Soares KTA, Viesser MV, Rzniski TAB, Brum EP. Eficácia de um protocolo de exercícios físicos em pacientes com insuficiência renal crônica, durante 0 tratamento de hemodiálise, avaliada pelo SF-36. Fisioter Mov. 2011; 24(1):133-40.

13. Corrêa LB, Oliveira RN, Cantareli FJS, Cunha LS. Efeito do treinamento muscular periférico na capacidade funcional e qualidade de vida nos pacientes em hemodiálise. J Bras Nefrol. 2009; 31(1):18-24

14. Torkington $\mathrm{M}$, MacRae $\mathrm{M}$, Isles $\mathrm{C}$. Uptake of and adherence to exercise during hospital haemodialysis. Physiotherapy. 2006; 92:83-7.

15. Soares A, Zehetmeyer $M$, Rabuske $M$. Atuação da Fisioterapia durante a Hemodiálise visando à qualidade de vida do paciente renal crônico. Rev Saúde UCPEL. 2007; 1(1):7-12

16. Ware JE, Kisinski MJ, Bjorner JB, Turner-Bowker DM, Gandek B, Maruish ME. SF-36v2 ${ }^{\circledR}$ Health Survey: Admnistration guide for clinical trial investigators. Lincoln RI: QualityMetric Incorporated; 2008.

17. Ciconelli RM, Ferraz MB, Santos W, Meinão I, Quaresma MR. Tradução para língua portuguesa e validação do questionário genérico de avaliação de qualidade de vida SF-36 (Brasil SF-36). Rev Bras Reumatol 1998; 39:14350.

18. Ritvo PG, Fischer JS, Miller DM, Andrews H, Paty DW, LaRocca NG. Multiple Sclerosis Quality of Life Inventory: a user's manual. New York: The Consortium of Multiple Sclerosis; 1997

19. QualityMetric. Disponível

em: https://www.qualitymetric.com.

20. Strand V, Singh JA. Improved health-related quality of life with effective disease-modifying antirheumatic drugs: evidence from randomized controlled trials. Am J Manag Care 2008;14:234-54

21. Lubeck DP. Patient-reported outcomes and their role in the assessment of rheumatoid arthritis. Pharmacoeconomics. 2004; 22:27-38.

22. Castro M, Caiuby AVS, Draibe AS, Canziani MEF. Qualidade de vida de pacientes com insuficiência renal crônica em Hemodiálise avaliada através do instrumento genérico SF-36. Rev Assoc Med Bras 2003; 49(3):245-9.

23. Cunha MS, Andrade V, Guedes CAV, Meneghetti CHZ, Aguiar AP, Cardoso AL. Avaliação da capacidade funcional e da qualidade de vida em pacientes renais crônicos submetidos a tratamento hemodialítico. Fisioter Pesq. 2009;16(2):155-60.

24. Martinez BB, Silva FM, Corrêa FC, Almeida L. Associação entre exercício físico e qualidade de vida de pacientes em hemodiálise. Rev Med (São Paulo). 2011;90(1):52-7.

25. Guedes KD, Guedes HM. Qualidade de vida do paciente portador de insuficiência renal crônica. Ciênc Saúde Colet 2012;5(1):48-53.

26. Peres CPA, Delfino VDA, Peres LAB, Kovelis D, Brunetto AF. Efeitos de um programa de exercícios físicos em pacientes com doença renal crônica terminal em hemodiálise. J Bras Nefrol. 2009;31(2):105-13.

\section{Endereço para correspondência:}

Clarissa Rios Lara

Rua das Pitangueiras $n^{\circ} 55$

Salvador/Bahia - CEP 40255-436

Telefone: +557188050590

E-mail: clarissarioslara@hotmail.com 